Abstract P0-0144 Table 1 Mean values (SD) of the study population, divided in two groups, according to insulin response. Mann-Whitney non parametric test for independent samples

\begin{tabular}{lllll}
\hline & All subjects & $\begin{array}{l}\text { Non insulin } \\
\text { resistance }\end{array}$ & $\begin{array}{l}\text { Insulin } \\
\text { resistance }\end{array}$ & \\
Parameters & $\mathbf{n}=\mathbf{2 6 8}$ & $\mathbf{n}=133$ & $\mathbf{n}=135$ & $\mathbf{p}^{*}$ \\
\hline BMI z-score & $2.21(0.39)$ & $2.16(0.41)$ & $2.25(0.36)$ & $0.019^{*}$ \\
Insulin (UI/ml) & $16.84(10.27)$ & $11.747(5.67)$ & $21.916(11.28)$ & $<0.001^{*}$ \\
Triglycerides (mg/dl) & $107.07(54.32)$ & $93.63(39.56)$ & $120.50(63.19)$ & $<0.001^{*}$ \\
HOMA index & $3.60(2.31)$ & $2.44(1.20)$ & $4.79(2.53)$ & $<0.001^{*}$ \\
TyG index & $8.33(0.47)$ & $8.20(0.41)$ & $8.47(0.49)$ & $<0.001^{*}$ \\
Triglycerides/HDL ratio & $2.42(1.69)$ & $1.20(1.09)$ & $2.86(2.03)$ & $<0.001^{*}$ \\
Waist/Height ratio & $0.59(0.06)$ & $0.58(0.06)$ & $0.59(0.05)$ & 0.303 \\
\hline
\end{tabular}

Results 135 subjects showed insulin resistance, with higher TyG index than those with normal insulin sensitivity $(\mathrm{P}<0.001)$ and higher Triglycerides/HL ratio $(\mathrm{P}<0.001)$ (Table).

In all the studied population a positive association between TyG index, HOMA index and Triglycerides/HDL ratio was found $(\mathrm{P}<0.001)$; in the non-insulin resistance group a positive association between TyG index and waist/height ratio was observed ( $p=0.03)$.

Conclusions TyG index is a good predictor of decreased insulin sensitivity also in paediatric ages and might be considered also a marker of cardiovascular risk considering the association with waist/height ratio and Triglycerides/HDL ratio.

\section{PO-0145 CELIAC DISEASE - THE EXPERIENCE OF A SINGLE CENTRE IN 29 YEARS}

G Lesanu, RM Vlad, O Dragnescu, 0 laru, M Stocklosa, I Tincu, R Smadeanu, C Becheanu, D Pacurar. Department of Pediatrics, "Grigore Alexandrescu" Emergency Children's Hospital, Bucharest, Romania

\subsection{6/archdischild-2014-307384.808}

Aims The study aimed to evaluate the epidemiological and clinical profile of the patients diagnosed with celiac disease in 'Grigore Alexandrescu' Children's Hospital over a 29 years period.

Methods We performed a retrospective study including 224 patients diagnosed with celiac disease, from January 1985 until December 2013. Three groups resulted. The first group included patients diagnosed from 1985 until 1995, before celiac serology was available, the second group patients diagnosed from 1996 until 2006 using qualitative antitissue-transglutaminase antibodies and the third group patients diagnosed from 2007 until 2013 using quantitative antitissue-transglutaminase antibodies and HLA DQ2/DQ8 typing. From the medical records we extracted age and clinical characteristics of patients at presentation.

Results The groups included 1.8, 13.8 and 7.7 patients/year. The mean age at diagnosis was $5.9 \pm 4.7,4.4 \pm 3.6$ and $4.4 \pm$ 3.5 years respectively. Gastrointestinal symptoms were described in $100 \%, 86.8 \%$ and $59.2 \%$, particularly chronic diarrhoea in $90 \%, 51.9 \%$ and $48.1 \%$. Twenty percent of patients in the first group presented with celiac crisis, only $0.6 \%$ in the second group and none in the third. Non-gastrointestinal symptoms were not observed in the first group and were reported in the second and third groups in $13.1 \%$ and $61.1 \%$.

Conclusion Since 2007 a decreasing tendency in the number of patients diagnosed/year was observed. Gastrointestinal symptoms were initially the sole clinical finding, particularly chronic diarrhoea, but in time patients presented with non-gastrointestinal symptoms or oligosymptomatic/asymptomatic celiac disease with an increasing frequency.

\section{P0-0146 PHENOTYPICAL FEATURES IN CHILDHOOD WILSON DISEASE: THE EXPERIENCE OF A HEPATOLOGY CENTRE FROM BUCHAREST}

D Pacurar, A Moraru, RM Vlad, I Andronie, G Lesanu, D Oraseanu. Department of Pediatrics, "Grigore Alexandrescu" Emergency Children's Hospital, Bucharest, Romania

\subsection{6/archdischild-2014-307384.809}

Background and aims Wilson's disease (WD) may have a polimorphic clinical picture and positive diagnosis can sometimes be difficult. The study aimed to analyse the clinical and laboratory characteristics of children with WD, diagnosed in the "Grigore Alexandrescu" Children's Hospital, Bucharest.

Methods The study included 23 patients diagnosed with WD between 1995 and 2013. From the medical records we extracted: age, sex, family history, clinical manifestations and laboratory parameters at diagnosis.

Results The mean age at diagnosis was 12.1 years. Sex ratio was male/female $=2.1 / 1$. Eight patients had family history of WD. The frequency of clinical signs was: hepatomegaly (47.8\%), ascites $(22 \%)$, jaundice $(22 \%)$, splenomegaly $(22 \%)$, esophageal varices $(15 \%)$. Neurological manifestations were encountered in 4 cases. Children aged 10-14 years presented the largest number of clinical and laboratory abnormal findings. Urinary excretion of cooper was increased in 22 patients. Elevated transaminases was a common finding. Serum ceruloplasmin was low in $74 \%$. Haematological abnormalities were: thrombocytopenia (26\%), leucopenia (4\%). Hepatic steatosis was found in $56 \%$, fibrosis in $18 \%$ and signs of portal hypertension in $11 \%$. Liver biopsy was performed in 8 cases, 6 presenting vacuolar lesions. Genetic testing was performed in 6 patients, 4 had heterozygote exon 8 mutation.

Conclusions WD is sometimes difficult to diagnose. Symptoms are more frequent in elder children and are dominated by liver disease with moderate cytolysis syndrome without liver failure; rarely neurological signs are associated. Family screening is extremely useful and allows precocious diagnosis in asymptomatic patients with early therapy initiation.

\section{PO-0147 NUTRITIONAL STATUS AND GROWTH PATTERN IN CHILDREN WITH CHRONIC LIVER DISEASE}

${ }^{1}$ ME Zaki, ${ }^{2}$ ME Eid, ${ }^{3}$ RM Sayed, ${ }^{4}$ MA Mohamed, ${ }^{1} \mathrm{HF}$ Ahmed. ${ }^{1}$ Biological Anthropology, National Research Centre, Cairo, Egypt; ${ }^{2}$ Institute of Postgraduate Childhood Studies, Ain Shams University, Cairo, Egypt; ${ }^{3}$ Pediatrics, Faculty of Medicine Cairo University, Cairo, Egypt; ${ }^{4}$ Medical Research Division, National Research Centre, Cairo, Egypt

\subsection{6/archdischild-2014-307384.810}

Background Malnutrition and growth retardation are important consequences in children with chronic liver disease (CLD). The aim of this study was to evaluate nutritional status and physical growth of children with CLD.

Subjects and methods Fifty children with CLD, recruited from the outpatient clinic of paediatric hepatology and from the paediatric hepatology department of Paediatric Hospital, Cairo University were enrolled in the study. Their mean age was 2.05 years, ranged from 0.5 to 5.75 years. Physical growth and nutritional status were assessed from the $\mathrm{Z}$ score of anthropometric parameters, using the Egyptian growth reference data. 